\title{
Semantic-context effects on word recognition: Influence of varying the proportion of items presented in an appropriate context
}

\author{
JAMES R. TWEEDY, ROBERT H. LAPINSKI, and ROGER W. SCHVANEVELDT \\ State University of New York, Stony Brook, New York 11794
}

\begin{abstract}
Several recent experiments have shown that an appropriate semantic context facilitates word recognition. Lexical (word/nonword) decisions about a word such as "nurse" are faster when it follows a related word such as "doctor". The present experiment examines the consequence of varying the proportion of semantically related adjacent words. The effect of semantic context is found to depend on the overall proportion of related word pairs. More facilitation occurs when there is a greater proportion of related word pairs. This finding contradicts theories of word recognition which account for context effects solely by postulating transient increases in the accessibility of only those words semantically related to the particular preceding stimuli encountered by the observer. An adequate theory must include an account of strategic or adaptive processes in which the past usefulness of contextual information modulates its influence in the word recognition process.
\end{abstract}

The influence of contextual factors on human information processing represents a common theme in several current areas of investigation. The context in which linguistic information is experienced importantly affects comprehension and memory. For example, Bransford and Johnson (1973) have shown that memory for complex linguistic material can be dramatically improved by providing contextual information which presumably aids in comprehending and organizing the material. Similarly, the encoding-specificity hypothesis (Martin, 1972; Tulving \& Thomson, 1973) and associated empirical work further attempt to incorporate contextual influences in theories about encoding and retrieving information. Contextual information is also influential in seemingly simpler situations, such as recognizing words.

Some recent experiments using a lexical-decision paradigm have provided some detailed information about the effects of semantic context on recognizing words. In the lexical-decision task, people judge whether various strings of letters are words or nonwords. By encouraging quick and accurate responses indicating such decisions, the effect of semantic context is assessed by the speed and accuracy of responses to a word when it follows related vs. unrelated words. For example, the word "nurse" is classified faster following a related word such as "doctor" than following an unrelated word such as "lamp."

The evidence suggests that the facilitative effect of an appropriate semantic context in the lexical-decision task results from increased efficiency in encoding processes. For example, comparable contextual facilitation is obtained for both positive and negative responses in the same task (Schvaneveldt \& Meyer, 1973). In addition, quite similar context effects occur for the same stimuli in tasks with markedly different response requirements, such as pronouncing letter strings (Meyer, Schvaneveldt, \& Ruddy, 1975). These findings suggest that processes involved in selecting and executing the appropriate response in the task are not speeded by presenting material in an appropriate semantic context. If they were, larger context effects would be observed in situations where more demanding response requirements result in generally longer reaction times (cf. Sternberg, 1969).

In contrast, alterations in the form of the visual stimulus do affect the magnitude of context effects. Visual noise has retarded the recognition of words in an inappropriate semantic context by approximately $120 \mathrm{msec}$, while retarding recognition of words in an appropriate semantic context by only about $90 \mathrm{msec}$ (Meyer et al., 1975). Apparently, the deleterious effects of the visual noise on encoding the stimulus are partially offset by an appropriate semantic context.

The conclusion suggested by these experiments is that benefits of semantic context apply quite generally to the recognition of words. They are not specific to the particular response requirements of the lexicaldecision task. This conclusion is further supported by the demonstration of facilitative semantic-context effects in other word recognition tasks, such as identifying spoken words in noise (Miller, Heise, \& Lichten 1951) and identifying tachistoscopically presented words (Tulving \& Gold, 1963). Evidently, the lexicaldecision task converges with a number of other information processing paradigms in indicating that the primary focus of benefits accruing from an appropriate semantic context is the encoding of the information present in the stimulus.

The experiment reported in the present paper was intended to inquire further into the precise mechanism underlying these contextual effects. A variety of theories 
attempt to account for context effects by postulating specific and transient increases in the accessibility of a portion of the observer's memory for word meanings. The "spreading excitation" theory suggested by Quillian (1967) and others (Collins \& Loftus, 1975; Meyer \& Schvaneveldt, 1971; Schvaneveldt \& Meyer, 1973) is an example of this type of theory. It postulates a semantically organized lexical memory. Accessing a particular item in lexical memory causes an automatic spread of activation to semantically related items, facilitating their encoding upon a subsequent encounter. The concept of spreading excitation implies that any processing facilitation for a particular letter string results from the residual activation produced by the recent recognition of some semantically related words. If only such specific factors are involved, a particular set of preceding words should always facilitate the recognition of a related word by the same amount.

The postulated transience of the semantic-context effect has received experimental support from two lexical-decision experiments reported by Meyer, Schvaneveldt, and Ruddy (Note 1). Interposing a nonword or an unrelated word between two highly related words substantially diminished the amount of facilitation provided by the preceding related word. In a second experiment, simply introducing an interstimulus delay of $4 \mathrm{sec}$ was sufficient to decrease the facilitation provided by the preceding word to half its original value.

The experiment reported here was designed to assess the extent to which the effect of a specific context provided by a particular sequence of preceding words is dependent on a general contextual factor: the potential usefulness of a context word as a predictor of the semantic identity of the word which follows it. Three independent groups of subjects were used, and each was exposed to exactly the same set of words and nonwords in the course of the session. A particular subject saw each item only once during the experiment. The major dependent variable was the likelihood that a word presented (e.g., doctor) would be followed by a semantically related word (e.g., nurse). In all conditions, subjects encountered some adjacent words that were semantically related. Subjects in the high-likelihood condition saw most of the words presented as pairs of this type (e.g., aunt-uncle, army-navy). Subjects in the low-likelihood condition saw the same set of words, but in most of the pairs the two words were unrelated in meaning (e.g., aunt-navy, army-uncle).

Evidence that this likelihood manipulation has no effect on the amount of facilitation produced by related words would support a variety of current theories, similar to the spreading excitation theory, which explain context effects in terms of specific and automatic increases in the accessibility of words which are semantically related to recently encountered words. Such specific contextual facilitation may occur whenever a semantic relationship exists between the word being recognized and some recently perceived word. Since an identical set of words is used in each condition, these specific relationships should provide equivalent overall facilitation for each group.

On the other hand, if the size of the context effect depends on the general reliability of the immediately preceding item as a predictor of the identity of the word which follows, then context effects should increase with the likelihood that the immediately preceding input is semantically related to the letter string being scrutinized. This result would indicate that the effects of specific semantic relationships between words being recognized are modulated by an adaptive process. This process would reflect the subject's use of the increased predictive potential of the semantic context in the highlikelihood condition. The influence of such an adaptive component in the recognition process can be assessed by determining the magnitude of the context effect as a function of the proportion of words which are presented as pairs of related words.

If an increase in the size of the context effect is produced by changing the proportion of related pairs presented, it could be obtained via an increase in the speed of responding to words in context, or by a decrease in speed for words out of context, or by some combination of these two effects. Following the terminology used by Posner and Snyder $(1974,1975)$, these two possible effects can be identified as the "benefit" and "cost," respectively, produced by changes in the probability variable. The relative magnitudes of the cost and benefit associated with changes in the likelihood variable may provide additional constraints on theoretical explanations of the facilitative effect of an appropriate semantic context.

\section{METHOD}

The subjects were 80 undergraduates from introductory psychology classes at the State University of New York at Stony Brook. Each subject was tested individually in a session that lasted approximately $30 \mathrm{~min}$. A session consisted of nine blocks of trials, each block containing 18 pairs of letter-string stimuli. Two thirds of the letter strings presented to each subject ( 24 of the 36 in each block) were common English words of six letters or less, selected from standard association norms (Palermo \& Jenkins, 1964; Bousfield, Cohen, Whitmarsh, \& Kincaid, Note 2). The remaining one-third of the letter strings were pronounceable nonwords produced by substituting an inappropriate letter in a common English word. These materials were almost identical to those used in previous lexical-decision experiments (e.g., Meyer \& Schvaneveldt, 1971).

The letter strings were presented on a cathode-ray display screen, which the subject observed in a darkened room from a distance of approximately $1.5 \mathrm{~m}$. The letter strings were printed left to right in uppercase letters approximately $80 \mathrm{~mm}$ high and $60 \mathrm{~mm}$ wide. The subjects responded by pressing one of two response keys: One labeled "yes" was pressed with the right index finger whenever the letter string was a word, and one labeled "no" was pressed with the left index finger whenever the letter string was a nonword. The presentation of a pair of letter strings was preceded by an orientation pattern which defined two rectangular areas in which the two letter strings of the pair 
were presented. This preexposure display was presented for $750 \mathrm{msec}$, then the first letter string appeared within the lefthand presentation area. The letter string remained visible until the subject responded. At this point, the characters of the first letter string were masked by the superimposition of nonsense characters, and the second letter string of the pair was displayed in the right-hand presentation area. Approximately $100 \mathrm{msec}$ elapsed between the subject's response to the first letter string and the onset of the second in each pair. Following the response to the second letter string in the pair, the entire display was erased and, following an interval of $500 \mathrm{msec}$, the preexposure display for the next pair of letter strings appeared.

Subjects were instructed to respond as quickly and accurately as possible. Reaction times were measured to the nearest millisecond from the onset of the letter string to the response. At the conclusion of each block, a feedback display indicated the number of correct responses in the block, the number which were too long (greater than $1,200 \mathrm{msec}$ ), and the number of errors.

Each block of 18 pairs of letter strings contained eight pairs in which both letter strings were words, four pairs in which a nonword was followed by a word, four pairs in which a word was followed by a nonword, and two pairs in which both letter strings were nonwords. In this arrangement, the probability that a letter string was a word, in either the first or second position within a pair, was $12 / 18$ or .67 . The conditional probability of a word following a word within a pair was $8 / 12$ or .67 . Thus, the lexical status of the first item provides no statistical information which might bias responses to the second item.

Three independent groups of subjects were used in the experiment. All groups were exposed to the same set of words and nonwords in the course of the session. The groups differed only in the proportion of cases where the two members of a word-word pair were semantically related. Each trial block contained eight word-word pairs. In the high-probability condition, Group H, seven of the pairs consisted of semantically related words. In the medium-probability condition, Group $M$, the words were related in four pairs and unrelated in four pairs. In the low-probability condition, Group L, only one of the eight word-word pairs in each block contained related words.

For all subjects the first block of letter strings was a practice block. Data from this portion of the experiment were not analyzed. Letter strings appearing in the practice block were identical for all groups, except that the proportion of pairs of related words was identical to the proportion used for that group during the remaining eight blocks of the session. Prior to the practice block, the subjects were instructed about the details of the task, and during the instructions each subject saw and responded to six pairs of letter strings (which never reappeared in the session).

The 8 word-word pairs presented in each of the remaining eight blocks were derived from a set of 64 highly related word pairs. For subjects in Group $\mathrm{H}, 8$ of these 64 word pairs were rendered unrelated by exchanging second members. For example, the unrelated pairs "doctor-light" and "lamp-nurse" are produced by exchanging the second members of the related word pairs "doctor-nurse" and "lamp-light." One of the eight unrelated pairs was presented, along with seven related pairs, in each of the eight final blocks of the experiment. For subjects in Group M, 32 of the 64 pairs were similarly rearranged and 4 of each type were presented in each of the eight blocks of trials. For subjects in Group L, 56 of the pairs were rearranged, and 7 of the rearranged pairs plus 1 related pair were presented in each block. The particular subset of word pairs selected for rearrangement varied from one subject to another within each group by a Latin square scheme which insured that the second member of each of the 64 word pairs was seen as part of a related pair by $87.5 \%$ of the subjects in Group $\mathrm{H}, 50 \%$ of the subjects in Group $M$, and $12.5 \%$ of the subjects in Group L.

The data of interest in this experiment are the reaction times to the second member of the 64 word-word pairs presented in the last eight trial blocks. Hereafter, these words will be called "critical items." Second members of the other pairs presented will be referred to as "noncritical items." The effects of semantic context are evaluated by comparing the average response time for making a lexical decision about a critical item in the related condition (nurse following doctor) with the average response time for a critical item in the unrelated condition (nurse following lamp). This comparison is somewhat difficult to make in Group $\mathrm{H}$ because only $12.5 \%$ of the subjects will see a particular critical item in the unrelated condition. A similar paucity of data exists for critical items in the related condition presented to subjects in Group L. To compensate for this, 32 subjects were tested in Groups $\mathrm{H}$ and $\mathrm{L}$, as opposed to 16 in Group $\mathrm{M}$.

\section{RESULTS}

The design of the experiment permits an assessment of the facilitation produced by an appropriate semantic context in each of three independent groups of subjects. This contrast can be based on 80 pairs of scores, each pair representing a given subject's average correct reaction time for critical items in the related and unrelated condition. The signed difference (unrelated minus related) of these two average latencies is an estimate of the size of the semantic context effect for that subject. Hereafter, this type of comparison will be referred to as a "subject" analysis. The same contrast can also be based on another set of 64 paired scores, each pair representing the average response time for a given critical item in the two different context conditions. Hereafter, this type of comparison will be referred to as an "item" analysis.

\section{Effect of the Context Variable}

The overall effect of semantic context is highly reliable statistically, regardless of whether a subject or item analysis is performed. The average processing facilitation, collapsing over the three different groups of subjects, is 55 msec. Evaluation of the effect of semantic context by subject analysis yields $F(1,77)=87.48$, $p<.01$. Average reaction time values in the item analysis are slightly different because of nonuniformities in the distribution of errors over subjects and items, but these discrepancies are quite small relative to the size of the context effect $(56 \mathrm{msec})$ which yields $F(1,63)=15.16, p<.01$.

\section{Interaction of the Context and Probability Variables}

For the purposes of this paper, the theoretically most interesting comparison is the evaluation of the changes in the size of the semantic-context effect produced by changing the proportion of related pairs. Inspection of Figure 1 reveals that the size of the context effect increases as the proportion of related pairs increases, indicating that subjects in Group $\mathrm{H}$ are relying more heavily on contextual information than are subjects in Group L. As with the previous comparisons, this interaction can be evaluated either in an item or a subject analysis. In both cases the interaction is statistically significant $[\mathrm{F}(2,126)=5.39, \mathrm{p}<.01$ and 


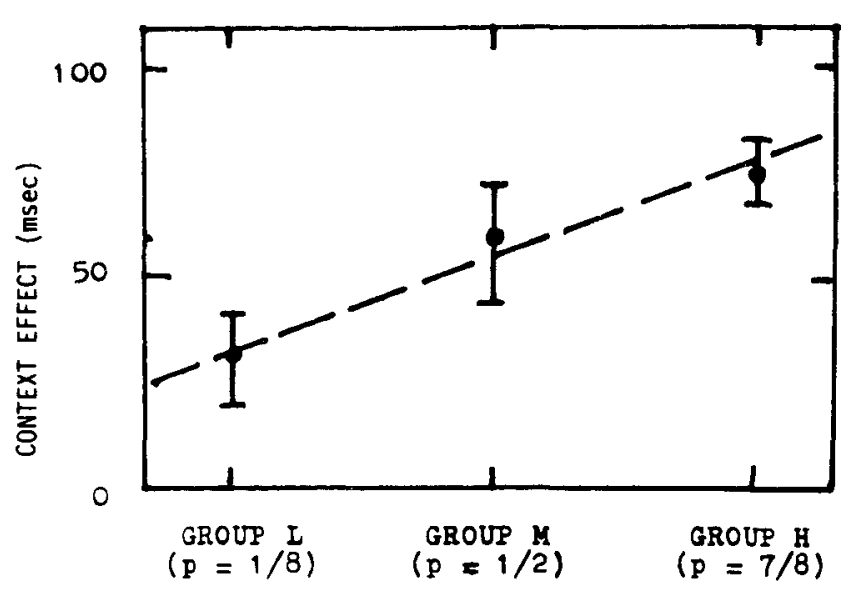

Figure 1. Size of the semantic-context effect for three different groups of subjects exposed to stimulus sequences which vary in the proportion (p) of semantically related word-word pairs.

$F(2,77)=5.50, p<.01$ in the item analysis and the corresponding subject analysis, respectively].

The dashed line in Figure 1 indicates the best-fitting linear regression for the average effect in each of the three different groups. The equation for this regression is:

\section{Context effect $(\mathrm{msec})=26+57.36(\mathrm{p})$}

where $p$ is the proportion of critical items that are presented in the related condition. Values for $p$ for Groups $L, M$, and $H$ in this experiment are $1 / 8,1 / 2$, and $7 / 8$, respectively. The linear component accounts for $98 \%$ of the variation among the three average values. The vertical bars represent the one standard error interval for each time average.

\section{Effect of the Probability Variable \\ Within Each Context Condition}

Figure 2 shows the average reaction time for correct responses to critical items in both the related and unrelated conditions for each group. The dashed lines indicate the best-fitting linear regression lines across groups for critical items in the related and unrelated context conditions. The equations for these regressions are:

$$
\begin{aligned}
& t_{\text {related }}(\mathrm{msec})=489-45.36(\mathrm{p}) \\
& t_{\text {unrelated }}(\mathrm{msec})=515+12.00(\mathrm{p})
\end{aligned}
$$

The substantial negative slope of the regression for responses to critical items in the related condition suggests that critical items appearing in an appropriate context are responded to more quickly as the value of $p$ increases. Using only data from the related context condition, this decrease across groups in reaction time for critical items is statistically significant in an item analysis $[F(2,126)=8.87, p<.01]$, but fails to achieve significance in subject analysis $[F(2,77)=1.73]$. The linear component accounts for $88 \%$ of the variation among the three average values.

Responses to critical items in the unrelated condition are evidently much less affected by the probability variable. The positive slope for the regression suggests that processing words out of context becomes somewhat more difficult as $p$ increases, but this effect is not statistically significant in the item analysis $[F(2,126)=2.11$, $\mathrm{p}>.10]$, or in the corresponding subject analysis $[\mathrm{F}(2.77)<1]$. The linear component accounts for $19 \%$ of the variation among the three average values.

\section{Effect of the Probability Variable on Noncritical Items}

Table 1 shows the average reaction time for correct responses for words following nonwords, nonwords following words, and nonwords following nonwords. Again, the slope of the best-fitting linear regression provides an estimate of the costs incurred in the processing of second items as a result of changes in the probability variable. The equations for these regressions are:

$$
\begin{aligned}
& t_{(w) n}(\mathrm{msec})=702+20.00(p) \\
& t_{(n) w}(\text { msec })=543+6.64(p) \\
& t_{(n) n}(m s e c)=710-8.00(p)
\end{aligned}
$$

The relatively large slope estimate for response times for nonwords which followed words suggests that the effects of the probability variable are greater when the initial letter string of the pair is a word. However, between-groups comparisons of average response times for all three types of pairs failed to approach statistical significance, so speculatinns about costs incurred for the

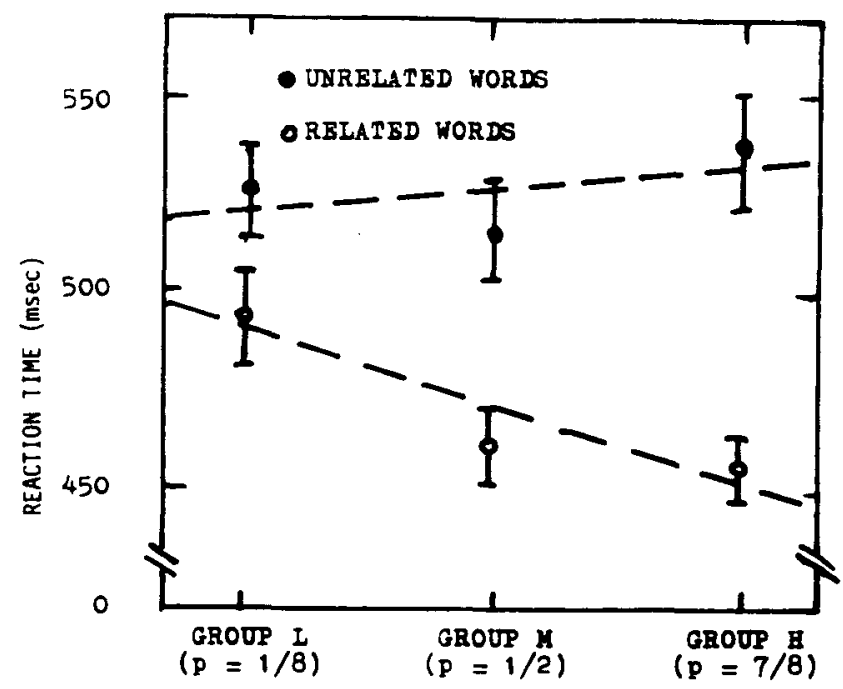

Figure 2. Mean reaction time for correct lexical decisions for critical items in related and un related context conditions for three different groups of subjects. 
Table 1

Average Correct Reaction Time in Milliseconds and Average Error Percentage (in Parentheses) for the Second Letter String of Each Pair Type in Each Probability Condition

\begin{tabular}{|c|c|c|c|c|}
\hline \multirow{2}{*}{$\begin{array}{l}\text { Pair } \\
\text { Type }\end{array}$} & \multirow{2}{*}{$\begin{array}{l}\text { Correct } \\
\text { Response }\end{array}$} & \multicolumn{3}{|c|}{ Group } \\
\hline & & $\mathrm{L}$ & $\mathbf{M}$ & $\mathrm{H}$ \\
\hline Related Word-Word & yes & $487 \quad(2)$ & 459 (1) & $453(1)$ \\
\hline Unrelated Word-Word & yes & 522 & $511(2)$ & 531 (2) \\
\hline Nonword-Word & yes & 549 (3) & $535 \quad(4)$ & 554 (5) \\
\hline Word-Nonword & no & $702(10)$ & $718(7)$ & 717 (9) \\
\hline Nonword-Nenword & no & $705(5)$ & 715 (5) & $699(6)$ \\
\hline
\end{tabular}

noncritical items which result from changing the proportion of related word-word pairs must remain tentative.

\section{Error Data}

The error rates for critical items in either the related or the unrelated condition were quite low for all three groups. Group 1 subjects responded incorrectly slightly more often than subjects in Groups $M$ or $H$, but no statistical comparisons involving error-rate data for either critical or noncritical items approached significance. Error rates for each item type in each of the three probability conditions are presented in Table 1 .

Because critical items in the related condition had slightly lower error rates than critical items in the unrelated condition for each of the three groups, the observed context effects cannot be attributed to a tendency of subjects to trade accuracy for speed in the related condition.

The generally higher error rates for "no" responses to nonwords, especially following "yes" responses to an initial word, may indicate a response-bias effect. However, the contrasts of primary interest all involve "yes" responses which follow "yes" responses to items in the word-word pairs, and such a response bias should not affect these comparisons.

\section{DISCUSSION}

The results indicate that while semantic context had a facilitative effect on word recognition in each of the three different probability conditions, substantially more facilitation was observed as the proportion of semantically related word pairs was increased. This interaction indicates that contextual effects cannot be solely attributed to specific changes in the ease of encoding words which are semantically related to previously encountered words. The recognition of "nurse" was facilitated in all conditions by a prior exposure to "doctor," and perhaps by other previously presented words as well. However, the beneficial consequences of these specific prior encounters are enhanced by situations in which the large number of related pairs presented makes the semantic identity of the previously presented item a potentially useful source of predictions about the identity of the letter string being scrutinized. The data suggest that contextual effects are at least partially produced by the observer's increased ability to take advantage of the predictability of the stimulus sequence when it contains frequent instances of semantically related word pairs.

To account for these data, an adequate theory might include an automatic component, such as the activation of representations of context-related words proposed by the spreading excitation theory, but it must also include some kind of adaptive process which increases the influence of context as its potential usefulness is increased. If contextual facilitation of word recognition is indeed partly automatic, it should be observed in situations in which the preceding sequence of letter strings presented to a subject never contained an instance of semantically related adjacent words. The present experiment did not include such a condition, but the data from Group $L$ indicate that substantial context effects $(35 \pm 8 \mathrm{msec})$ do exist even when the proportion of related pairs is low $(p=1 / 8)$. In a linear extrapolation from the data of the present experiment, the estimated size of the context effect when $p=0$ is $26 \mathrm{msec}$ (see Figure 1). Thus, the available evidence is compatible with the existence of an automatic component, although a more direct test requires an experiment where the observer encounters semantically related adjacent words only after experiencing many unrelated word pairs.

The adaptive component of the process could be conjoined with an automatic component in a variety of ways. One example of such a two-process theory is that proposed by Posner and Snyder (1974). Their automatic component results from activation of the memory representations of context-related words by a process analogous to spreading excitation. The process benefits recognizing related words without any cost for recognizing unrelated words. Their strategic component reflects the deployment of a person's conscious attention. Since attentional capacity is limited, benefits realized by attending to memory representations of related words should be accompanied by costs in recognizing unrelated words.

In the present experiment, increasing the proportion of related pairs produced faster recognition of words in context, but recognizing words out of context was not appreciably slower. There is, however, reason to expect small costs for unrelated words relative to the benefits for related words. Because the set of alternatives related to the context is small, substantial benefits from focusing attention on them could be obtained with only a slight decrease in the availability of attention for any item in the much larger set of alternatives that are unrelated to context. In contrast, Posner and Snyder (1974) observed considerable costs in their experiments, but their data come from identity judgment tasks using single-letter stimuli. Since the pool of stimulus alternatives in such experiments is small (the 26 letters of the alphabet) relative to the set of potential stimuli in the lexical-decision task, it is not surprising that Posner and 
Snyder's estimates of costs and benefits were more nearly symmetrical than those obtained in this experiment.

In conclusion, the results of the experiment support theories of word recognition that contain an adaptive component which allows the subject to rely more heavily on the context in which a word is presented when that context is more likely to provide a basis for predicting the identity of the word being presented. Evidence that the facilitation provided by an appropriate semantic context may also contain an automatic component rests on the nonzero intercept value of the linear function relating context facilitation and the proportion of stimuli presented in context. While the fit of this function was quite good over the three levels of the probability variable tested in the experiment, extremely low levels may not lie on the estimated function. A more direct test of the effects of context in the $p=0$ condition would be desirable. The evidence for an adaptive component is more compelling, yet the data do not allow any clear inferences about the source of this effect. The processes of analyzing sensory information, retrieving information from lexical memory, and response execution are all possible loci of the effect. Current theories, too, tend to be noncommital about this issue. Previous work (e.g., Meyer et al., 1975) has led to the conclusion that encoding processes are affected by local context. Encoding may also be affected by more general contextual factors, but more research is required to resolve the issue.

\section{REFERENCE NOTES}

1. Meyer. D. E., Schvaneveldt, R. W., \& Ruddy, M. G. Activation of lexical memon. Paper presented at the meeting of the Psychonomic Society, St. Louis, November 1973.

2. Bousfield, W. A., Cohen, B. H., Whitmarsh, G. A., \& Kincaid, W. D. The Connecticut tree association norms (Report No. 35). Department of Psychology, University of Connecticut. Storrs, 1961.

\section{REFERENCES}

Bransford, J. D., \& Johnson, M. K. Considerations of some problems of comprehension. In W. G. Chase (Ed.), Visual information processing. New York: Academic Press, 1973.

Collins, A. M., \& Loftus, E. F. A spreading-activation theory of semantic processing. Psychological Review, 1975, 82. 407.423.

MARTIN, E. Stimulus encoding in learning and transfer. In
A. W. Melton \& E. Martin (Eds.), Coding processes in human memory. Washington, D.C: Winston, 1972.

Meyer. D. E.. \& Schyaneveldt, R. W. Facilitation in recognizing pairs of words: Evidence of a dependence between retrieval operations. Joumal of Experimental Psychology, $1971,90,227-234$.

Meyer. D. E., Schvaneveldt, R. W., \& Ruddy, M. G. Loci of contextual effects on visual word recognition. In P. M. A. Rabbit \& S. Dornic (Eds.), Attention and performance $V$. New York: Academic Press, 1975.

Miller, G. A., Heise, G. A., \& Lichten, W. The intelligibility of speech as a function of the context of the text materials. Journal of Experimental Psychology, 1951, 41, 329-335.

PAlermo, D. S., \& Jenkins, J. J. Word association normsgrade school through college. Minneapolis: University of Minnesota Press, 1964.

Posner, M. I., \& S SYder, C. R. R. Facilitation and inhibition in the processing of signals. In P. M. A. Rabbitt \& S. Dornic (Eds.), Attention and performance $V$. New York: Academic Press, 1974

Posner, M. I., \& SNyder, C. R. R. Attention and cognitive control. In $\mathrm{R}$. Solso (Ed.), Information processing and cognition: The Loyola symposium. Potomac, Md: Lawrence Erlbaum, 1975.

Quillian, M. R. Word concepts: A theory and simulation of some semantic capabilities. Behavioral Science, 1967. 12. $410-430$.

SchVaneveldt, R. W., \& Meyer, D. E. Retrieval and comparison processes in semantic memory. In $\mathbf{S}$. Kornblum (Ed.), Attention and performance IV. New York: Academic Press. 1973.

STERnBERG, S. The discovery of processing stages: Extensions of Donders' method. In W. G. Koster (Ed.), Attention and performance II. Amsterdam: North Holland, 1969.

Tulving, E. \& \& Gold, C. Stimulus information and contextual information as determinants of tachistoscopic recognition of words. Journal of Experimental Psychology, 1963, 66. 319-327.

Tulving, E., \& Thомson, D. M. Encoding specificity and retrieval processes in episodic memory. Psychological Review, 1973, 80, 352-373.

\section{NOTE}

1. In a subject analysis, comparisons of different probability levels are evaluated with an error term which includes betweensubjects variation. This tends to produce $F$ ratios which are smaller than the corresponding $F$ ratios obtained from an item analysis. Since the same critical items appear in each of the three groups, item variability is not included in the error term of an item analysis $\mathrm{F}$ ratio. In contrast, context condition is a withinsubjects variable and can be tested as a within effect in both a subject and an item analy sis.

(Received for publication January 6, 1976; revision received June 9,1976 .) 